\title{
The Effect of Adding Sufentanil to 0.5\% Hyperbaric Bupivacaine on Duration of Brachial Plexus Blockade in Chronic Opium Abusers: a Randomized Clinical Trial
}

\author{
Omid Azimaraghi ${ }^{1}$; Seyed Mojtaba Marashi ${ }^{1}$; Noushin Khazaei ${ }^{2}$; Sarah Pourhassan ${ }^{3}$; Ali \\ Movafegh ${ }^{1, *}$ \\ ${ }^{1}$ Department of Anesthesiology, Pain and Critical Care, Dr. Shariati Hospital, Tehran University of Medical Sciences, Tehran, Iran \\ ${ }_{2}^{2}$ Department of Anesthesiology, Shahid Beheshti University of Medical Sciences, Tehran, Iran \\ ${ }^{3}$ Department of Medicine, Tabriz University of Medical Sciences, Tabriz, Iran \\ ${ }^{*}$ Corresponding author: Ali Movafegh, Department of Anesthesiology, Pain and Critical Care, Dr. Shariati Hospital, Tehran University of Medical Sciences, Tehran, Iran. Tel: \\ +98-9123021389, Fax: +98-88220032, E-mail: Movafegh@tums.ac.ir
}

Received: July 12, 2014; Revised: October 4, 2014; Accepted: November 11, 2014

\begin{abstract}
Background: Anesthesia induction in patients with current substance abuse can be a challenge for anesthesiologists.
Objectives: This study aimed to evaluate the effect of adding Sufentanil to Bupivacaine on duration of brachial plexus nerve block.

Patients and Methods: One hundred and twenty patients with (Groups C and D) and without (Groups A and B) a history of opium abuse (60 in each group) scheduled for elective upper extremity procedures were randomly assigned to either receive $30 \mathrm{~mL}$ bupivacaine alone (Groups A and C) or in combination with additional $10 \mu \mathrm{g}$ sufentanil (Groups B and D). An ultrasound-guided technique was applied to perform upper extremity brachial plexus blockade. The onset and duration of sensory and motor blocks were recorded and compared between the four groups.

Results: The duration of sensory and motor block were significantly less in Group C (537.0 \pm 40.1 minutes, $479.0 \pm 34.8$ minutes) and the longest duration of sensory and motor block was observed in group B (705.0 \pm 43.8 minutes, $640.0 \pm 32.5$ minutes). The duration of sensory and motor block in Group B (705.0 \pm 43.8 minutes, $640.0 \pm 32.5$ minutes) was longer and statistically higher than group A (619.5 \pm 48.0 minutes, $573.2 \pm 31.5$ minutes), the same trend was observed in group D (598.6 \pm 53.2 minutes, $569.3 \pm 39.9$ minutes) over group $C$ ( $537.0 \pm$ 40.1 minutes, $479.0 \pm 34.8$ minutes $)(\mathrm{P}<0.001$, one-way ANOVA).

Conclusions: The length of sensory and motor blockade is shorter in chronic opioid abusers. Adding $10 \mu \mathrm{g}$ sufentanil to hyperbaric bupivacaine in opium abusers lengthened the sensory and motor block duration.
\end{abstract}

Keywords: Substance-related Disorders; Nerve Block; Bupivacaine; Sufentanil

\section{Background}

Psychoactive substances are consumed for thousands of years. Illicit demand for opium and its derivatives is continuing to increase, notably in young males living in urban areas (1). Although established illicit drug markets in many developed countries have shown signs of stabilization, growth of drug use seems to continue in many developing countries (1). Globally, it was estimated that in 2010 between 153 and 300 million people aged 15-64 years (3.4-6.6 percent of the world's population in that age group) had used an illicit substance at least once (1).

In Iran, opioids are the primary drugs of abuse in the society $(2,3)$. Determining a definite estimate of prevalence and incidence of substance abuse is not possible due to social stigmatization along with legal restrictions (4). Sensory and motor blockade behaviors of brachial plexus block in long-term chronic opioid users has not been previously studied thoroughly. Previously, the duration of spinal anesthesia with hyperbaric bupivacaine in chronic opium abusers undergoing lower extremity orthopedic surgery was studied (5). It was shown that the duration of sensory block was much shorter in chronic opium abusers compared to non-abusers. The hypothesis of our study was that the duration of brachial plexus block in chronic opium abusers is shorter than non-abuser ones. We further hypothesized that adding sufentanil can increase blockade time in chronic opium abusers and overcome this problem in procedures needing regional blockade.

\section{Objectives}

In the current study, we evaluated the effect of adding sufentanil to bupivacaine $0.5 \%$ in opium abusers versus subjects without a history of opium abuse by measuring sensory and motor blockade in four groups of patients. Secondary, we evaluated the effect of adding sufentanil on the onset of blockade. 


\section{Patients and Methods}

The Institutional Ethics Committee of Tehran University of Medical Sciences, Tehran, Iran, approved the study protocol. The study was explained thoroughly to participants, and an informed written consent was obtained from all patients. Corresponding IRCT number for the current study is IRCT201209165140N5. One hundred and twenty subjects (60 opium abusers and 60 without a history of opium abuse) American Society of Anesthesiologist (ASA) physical status class I and II, male, current smokers, aged between 18-60, scheduled for elective upper limb orthopedic surgery under supraclavicular nerve block in Shariati Hospital affiliated to Tehran University of Medical Sciences were enrolled in this randomized, double-blinded clinical trial.

Chronic opium abuse was defined as recurrent and continuous daily consumption of 1 - 2 grams of opium via inhalation route for at least one year without a cessation until the day of operation based on patients' report. To rule out opium use in control groups and confirm opium use in the study group, in all patients, a rapid opiate urine test was performed. None of the patients had any intention to stop opium use before the operation and all the patients continued using their typical inhaled opium until the day of operation in the preoperative visit. Patients were given their daily doses of inhaled opium on the day of operation.

Patients with any contraindications to supraclavicular nerve block, patients with addiction to any substance other than opium and cigarettes, and patients with known history of cardiac, respiratory, or psychological diseases were not entered in the study. All required drugs were prepared by an anesthetist not involved in the administration or observation of patients; thus, both anesthesiologist and patients were blinded to group assignment. The anesthesiologist who performed the nerve block and documented sensory levels was also blinded to patient groups.

The patients were first assigned to two different groups based on opium abuse history, then patients in each group were randomly assigned to either bupivacaine plus saline or bupivacaine plus sufentanil based on a computer generated list. The study groups were defined as follows: Group A $(n=30)$ had no history of chronic opium use and received $30 \mathrm{~mL}$ hyperbaric bupivacaine along with $2 \mathrm{~mL}$ saline as placebo. Group B $(\mathrm{n}=30)$ had no history of opium use and received $30 \mathrm{~mL}$ hyperbaric bupivacaine along with $2 \mathrm{~mL}$ sufentanil $(10 \mu \mathrm{g}, \mathrm{n}=30)$. Group C $(\mathrm{n}=30)$ had a positive history of chronic opium use and received $30 \mathrm{~mL}$ hyperbaric bupivacaine along with $2 \mathrm{~mL}$ saline as placebo. Lastly, group $\mathrm{D},(\mathrm{n}=30)$ had a positive history of chronic opium abuse and received $30 \mathrm{~mL}$ hyperbaric bupivacaine along with $2 \mathrm{~mL}$ sufentanil (10 $\mu \mathrm{g})$. The consort flow diagram is shown in Figure 1.

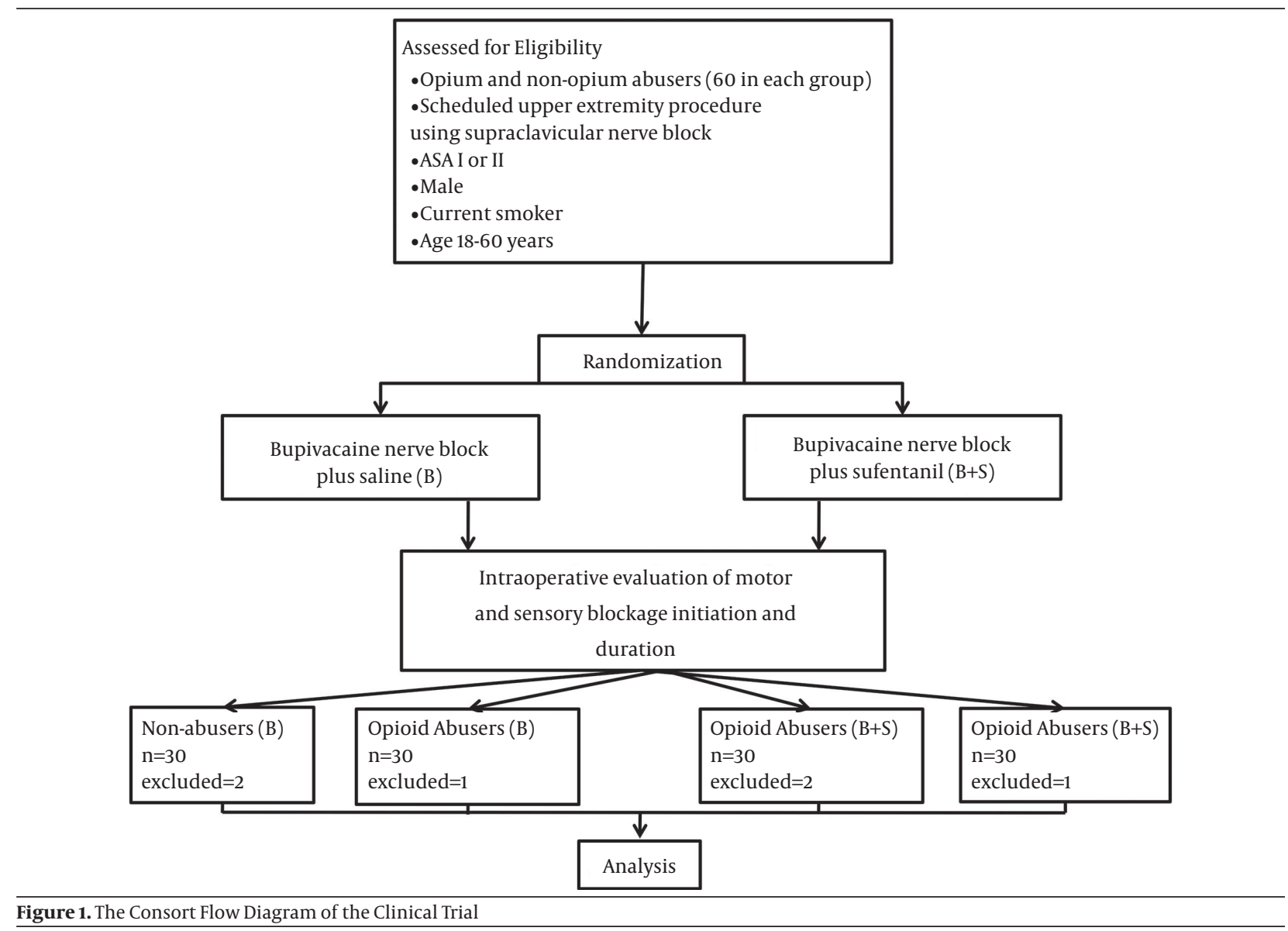


On arrival to the operating room, standard monitoring was applied (pulse oximetry, noninvasive arterial blood pressure and electrocardiography). Supplemental oxygen was delivered via face mask at a rate of $3-5$ lit/minute. After, an 18-gauge IV catheter was inserted in a peripheral vein on the dorsum of non-injured hand and $1 \mathrm{mg}$ of midazolam was administered.

To perform supraclavicular block, patients were positioned supine, with the head turned to contralateral side, rotated to an angle of 45 degree from midline and the arm was placed at the side. After skin sterilization and preparation, the probe was covered with sterile dressing. The subclavian artery was palpated and visualized using SonoAce X8 ultrasound machine (Samsung Medison, South Korea) with a linear probe with a frequency range of 5-12 MHz. Then the skin was anesthetized by subcutaneous injection of 3 $\mathrm{mL}$ 1\% lidocaine. Next, under ultrasonographic guidance, a 22-gauge, $5 \mathrm{~cm}$ insulated Sprotte needle was advanced along the long axis of the probe from lateral to medial direction, the supraclavicular plexus sheath was penetrated by an in-plane technique. When the needle reached the brachial plexus cluster, correct positioning of needle tip was demonstrated with ultrasound. For precise localization of the nerve cluster, using Plexygon nerve stimulator (Vygon Vet, UK), stimulation began at a frequency of $1 \mathrm{~Hz}$, with a duration of $0.1 \mathrm{~ms}$. The intensity of stimulating current was initially set to deliver 1 - $2 \mathrm{~mA}$ and was then gradually decreased. The correct site of needle was confirmed when output current 0.2 - $0.4 \mathrm{~mA}$ still elicited a distal motor response. Subsequently, the needle was redirected and advanced to the most superficial portion formed by brachial plexus trunks and divisions in the lateral side of cluster and the remaining $15 \mathrm{~mL}$ local anesthetic was injected after negative aspiration was performed. During injection of every 5.0 - 6.0 mL, negative aspiration was performed to avoid intravascular injection. Subsequently, the needle was redirected and advanced to the most superficial portion.

Preoperative pain management protocol was the same for all patients. The patients received intermittent (every 6 hours) intravenous Apotel $(15 \mathrm{mg} / \mathrm{Kg}$ ) (Intravenous Paracetamol $1000 \mathrm{mg} / 6.7 \mathrm{~mL}$, UNI-PHARMA S.A.) if the VAS score for pain was higher than three. Diclofenac suppository was administered to patients who had pain despite intravenous Apotel administration. In the case of block failure of any nerve distributions (i.e. if the patient felt pain in those regions), the patient was excluded from the study, even when the block was adequate to perform the operation.

Sensory and motor block was checked continuously after completion of injection until complete sensory and motor block and every 15 minutes following the end of operation. A pinprick test was used and compared with the same stimulation on the contralateral hand. The sensory block was assessed by a verbal rating scale from $100 \%$ (normal sensation) to 0 (no sensation). The motor block was evaluated by performing thumb abduction (radial nerve), thumb adduction (ulnar nerve), thumb opposi- tion (median nerve) and elbow flexion (musculocutaneous nerve). A modification of the Lovett rating scale was used to assess the motor block as follows: 0 (complete paralysis), 1 (almost complete paralysis), 2 (pronounced mobility impairment), 3 (slightly impaired mobility), 4 (pronounced reduction of muscular force), 5 (slightly reduced muscular force) and 6 (normal muscle force).

The sensory and motor block onset time was defined as the time between the end of the last injection and complete absence of pinprick response and complete paralysis (Lovett rating scale $=0$ ) in all nerve distributions. The duration of sensory block was defined as the time interval between complete sensory block (complete absence of pinprick response) and the first postoperative pain. The motor block duration time was defined as the time interval between complete paralysis (Lovett rating scale $=$ 0 ) and complete recovery (Lovett rating scale $=6$ ). It was estimated that a minimum of 30 patients in each group would be required to have a $95 \%$ power of detecting a 50-minute sensory block time difference at a significance level of 0.05 . Normal distribution of age, height, weight, surgery time, onset time and duration of sensory and motor block were evaluated using the Kolmogorov-Smirnov test. All tested variables followed a normal distribution. Height, weight, age, onset time, operation time and duration of sensory and motor block were compared between the four groups by one-way analysis of variance (ANOVA) and Tukey post hoc tests. The ASA physical status and gender were compared using chi-square test and Fisher's exact test. Two-tailed $\mathrm{P}<0.05$ was considered significant.

\section{Results}

\subsection{Patients' Characteristics}

Six patients were excluded from the study due to unsuccessful blocks. There were no statistically significant differences in unsuccessful blocks between the groups. The patients' mean weight, height, age, type and duration of surgeries (Table 1) and ASA physical status classes were the same in the four groups. All patients in groups $C$ and $D$ had positive results for rapid urine test and all the patients in groups $A$ and $C$ had negative results for urine exam.

\subsection{Primary and Secondary Outcomes}

The duration of sensory and motor block time was shortest in Group C (537.0 \pm 40.1 minutes, $479.0 \pm 34.8$ minutes) and the longest duration of sensory and motor block was observed in group B (705.0 \pm 43.8 minutes, $640.0 \pm 32.5$ minutes). The duration of sensory and motor block in group B (705.0 \pm 43.8 minutes, $640.0 \pm 32.5$ minutes) was longer and statistically higher than group A (619.5 \pm 48.0 minutes, $573.2 \pm 31.5$ minutes $)$, the same trend was observed in group D (598.6 \pm 53.2 minutes, $569.3 \pm 39.9$ minutes $)$ versus group $C(537.0 \pm 40.1 \mathrm{~min}-$ utes, $479.0 \pm 34.8$ minutes $)(\mathrm{P}<0.001$, one-way ANOVA) (Figure 2). There were no significant differences in onset 
Azimaraghi $O$ et al.

\begin{tabular}{|c|c|c|c|c|}
\hline Variable & Group 1 & Group 2 & Group 3 & Group 4 \\
\hline Age, $y^{c}$ & $37.4 \pm 9.2$ & $32.7 \pm 8.3$ & $35.3 \pm 7.3$ & $36 \pm 14.4$ \\
\hline Weight, kg ${ }^{\mathrm{d}}$ & $81.4 \pm 9.2$ & $78.0 \pm 7.4$ & $73.5 \pm 2.9$ & $73.9 \pm 8.3$ \\
\hline Height, cm $^{\mathrm{d}}$ & $172.4 \pm 9.2$ & $173.1 \pm 8.5$ & $174.6 \pm 5.3$ & $169.4 \pm 7.9$ \\
\hline Duration of operation, min $^{d}$ & $109 \pm 28.9$ & $97.3 \pm 19.9$ & $98.3 \pm 25.2$ & $101.3 \pm 24.7$ \\
\hline $\operatorname{ASA}(\mathbf{I} / \mathbf{I I})$ & $17 / 13$ & $12 / 18$ & $13 / 17$ & $16 / 14$ \\
\hline
\end{tabular}

a Abbreviation: ASA, American society of anesthesiologist.

$\mathrm{b}$ Data are presented as mean \pm SD.

c Sample size was 30 .

$\mathrm{d}$ There was no significant difference between the groups .

Figure 2. Duration and Onset of Sensory and Motor Nerve Blocks

Onset and Duration of Sensory arid Motor Block time

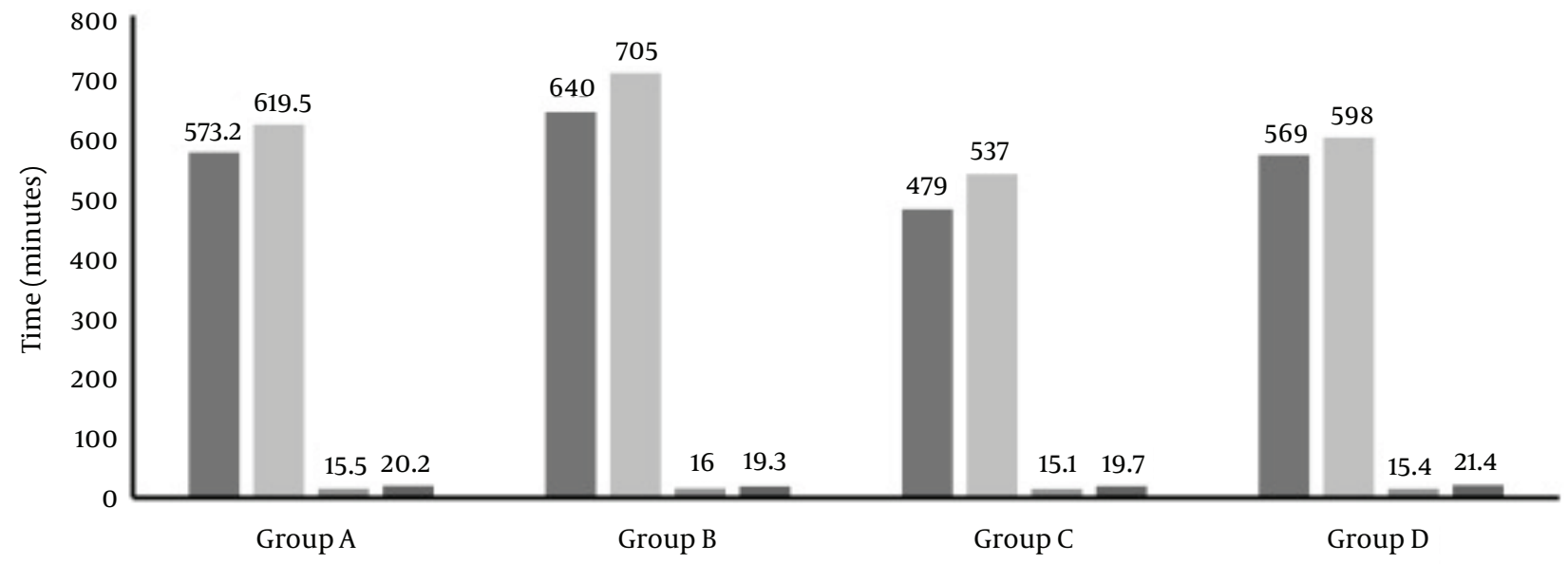

mDuration of motor Block $=$ Duration of Sensory Block $\equiv$ Onset of Sensory Block $\approx$ Onset of Motor Block

Group A had no history of chronic opium use and received $30 \mathrm{~mL}$ hyperbaric bupivacaine along with $2 \mathrm{~mL}$ saline as placebo. Group B had no history of opium use and received $30 \mathrm{~mL}$ hyperbaric bupivacaine along with $2 \mathrm{~mL}$ sufentanil. Group C had a positive history of chronic opium use and received $30 \mathrm{~mL}$ hyperbaric bupivacaine along with $2 \mathrm{~mL}$ saline as placebo. Group D had a positive history of chronic opium use and received $30 \mathrm{~mL}$ hyperbaric bupivacaine along with $2 \mathrm{~mL}$ sufentanil $(10 \mu \mathrm{g})$.

of sensory and motor block in Groups A, B, C and D (Tukey post hoc test). Interestingly, the duration of sensory and motor block time between group A and D was not statistically significant. The sensory and motor onset times were not statistically different between the groups.

\section{Discussion}

The present study demonstrated that both the sensory and motor block times were shorter in chronic opium abusers compared to non-abusers. Furthermore, adding $10 \mu \mathrm{g}$ of sufentanil to the local anesthetic solution in ultrasound guided brachial plexus block, prolonged both the sensory and motor block times in chronic opium abusers and non-abusers. No changes were observed in the onset of sensory or motor block times in any of the groups.

Dealing with patients with substance abuse could be a challenge for anesthesiologists. Acute postoperative pain management in patients with current opioid con- sumption is a challenge for anesthesiologists, as well as the best plan for a smooth course through anesthesia. Regional anesthesia has been long proposed for opioid dependent patients and suggested by many anesthesiologists as a respectable plan of both anesthesia and postoperative pain management for surgeries on extremities in such patients $(6,7)$. Nevertheless, very few studies actually examined clinical outcome of regional anesthesia in opioid abusers. After a systemic search in well-known databases, very few data were found regarding the behavior of peripheral nerve blocks in chronic opium abusers.

Previously, a shorter duration of sensory and motor blockade has been reported in chronic opium abusers compared to non-abusers. It was proposed that a crosstolerance may exist between local anesthetics and opioid compounds at the level of spinal neurons (5). In another study conducted by the same team, the effect of intrathecally administration of lidocaine in spinal anesthesia 
was studied in chronic opioid users with similar outcomes, including shorter sensory and motor blockade during spinal anesthesia in chronic opium abusers (8). To lengthen this shortened duration in chronic opium abusers, fentanyl, midazolam and ketamine have been proposed as an adjuvant (9-12).

In more recent studies, it was proposed that adding sufentanil to intrathecal bupivacaine may prolong the duration of analgesia and facilitate the spread of sensory block (13). Studying patients with a history of drug abuse has its own challenges. Cross-addiction or polysubstance abuse renders studies on such populations. Patients with substance abuse may also be dependent on other drugs such as benzodiazepines. Apart from used substances by people, definitions such as dependency, abuse and tolerance used by health professionals are not robust enough to allocate such populations into clear defined groups.

Currently, the Diagnostic and Statistical Manual of Mental Disorders, 4th Edition, Text Revision, also known as DSM-IV-TR, includes all currently recognized mental health disorders and thus used by mental health professionals to describe features of a given mental disorder and indicate how the disorder can be distinguished from others and similar problems. Nonetheless, based on the terms used in the mentioned criteria, it is not possible to clearly distinguish people for clinical practice in anesthesia or pain management, even though these patients need more individualized attention. Therefore, there is a need for special diagnostic criteria, apart from the DSMIV criteria, to differentiate patients regarding their needs for pain and anesthesia management.

In the present study, urine test was performed to evaluate whether patients had used opioids, but it had its own shortcomings. The exact daily dosage of opium consumption in each patient and concentration of effective alkaloids in used opium were unknown. Besides, cultural issues, legal restrictions and personality traits make such investigations more difficult $(2,3,14)$. Patients may be concerned about the social stigma that comes with addiction, and therefore not acknowledge their problem, an issue that is a great obstacle to such researches in $\operatorname{Iran}(2,3)$. To help overcome this, we had to do a urine test in all patients to detect abusers. In the present study, females were not included because of the mentioned social stigma that comes with addiction and cultural issues in Iran.

The mechanism of modification of opioid effect in drug abusers was not the aim of the current study, but this effect of lower duration of peripheral nerve blocks may be partially explained by down-regulation of opioid receptors or a cross tolerance between opioids and local anesthetic receptors. Despite the fact, it is necessary to perform more investigations to clearly describe pathways altered or modified in chronic opioid abusers (14-16).

Opioids analgesic effect through the central nervous system (CNS) has been studied previously thoroughly; however, evidence exists that opioid anti-nociception can be initiated by activation of opioid receptors located out- side the CNS; one of the earliest reports was that of Wood (15). Previous studies indicated that a large amount of analgesic effects produced by systemically administered opioids could be mediated by peripheral opioid receptors (17-19). Undoubtedly, opioid agonists acting peripherally would be more attractive for their lack of central adverse effects and only showing typical adverse effects of non-steroidal anti-inflammatory drugs (20). Mechanism of action in peripheral opioid system needs to be translated into clinical practice. Considering the fact that the relation between pain perception and substance abuse is multifactorial, further studies are needed to understanding the underlying mechanisms.

In this study, our aim was to eliminate all possible confounding factors. The same anesthesiologist administered all blocks, and one anesthesiologist observed and collected all data. A potential line of research could be perioperative pain management in patients with current substance abuse. We are currently studying different management strategies for optimum operative and perioperative pain management in patients with current substance abuse.

In conclusion, both the sensory and motor block times were shorter in chronic opium abusers compared to nonabusers. Therefore, adding $10 \mu \mathrm{g}$ sufentanil to the local anesthetic solution in ultrasound guided brachial plexus block prolonged both the sensory and motor block times in chronic opium abusers and non-abusers.

\section{Authors' Contributions}

Omid Azimaraghi contributed in study design, data acquisition, data analysis and manuscript drafting. Sarah Pourhassan contributed to data analysis and manuscript drafting. Seyed Mojtaba Marashi and Ali Movafegh contributed to study design, monitoring the study and critical revision of the manuscript.

\section{Funding/Support}

The funding organization is a public institution and had no role in the design and conduct of the study, collection, management and analysis of data or preparation, review and approval of the manuscript.

\section{References}

1. United Nations Office on Drugs and Crime.. World drug report 2012.: United Nations Publications; 2012.

2. Mokri A. Brief overview of the status of drug abuse in Iran. Arch Iranian Med. 2002;5(3):184-90.

3. Moharreri MR. General view of drug abuse in Iran and one-year report of outpatient treatment of opiate addiction in the city of Shiraz. NIDA Res Monogr. 1978(19):69-81.

4. Day C, Nassirimanesh B, Shakeshaft A, Dolan K. Patterns of drug use among a sample of drug users and injecting drug users attending a General Practice in Iran. Harm Reduct J. 2006;3:2.

5. Dabbagh A, Dahi-Taleghani M, Elyasi H, Vosoughian M, Malek B, Rajaei S, et al. Duration of spinal anesthesia with bupivacaine in chronic opium abusers undergoing lower extremity orthopedic surgery. Arch Iran Med. 2007;10(3):316-20.

6. Rapp SE, Ready LB, Nessly ML. Acute pain management in pa- 
tients with prior opioid consumption: a case-controlled retrospective review. Pain. 1995;61(2):195-201.

7. Mitra S, Sinatra RS. Perioperative management of acute pain in the opioid-dependent patient. Anesthesiology. 2004;101(1):212-27.

8. Vosoughian M, Dabbagh A, Rajaei S, Maftuh H. The duration of spinal anesthesia with $5 \%$ lidocaine in chronic opium abusers compared with nonabusers. Anesth Analg. 2007;105(2):531-3.

9. Gupta S, Dev K, Katyal S, Kathuria S. Intrathecal fentanyl with $0.5 \%$ bupivacaine heavy in chronic opium abusers. South Afr J Anaesth Analg. 2012;18(2)

10. Safari F, Dabbagh A, Sharifnia M. The effect of adjuvant midazolam compared with fentanyl on the duration of spinal anesthesia with $0.5 \%$ bupivacaine in opium abusers. Korean J Anesthesiol. 2012;63(6):521-6.

11. Dahi-Taleghani M, Fazli B, Ghasemi M,Vosoughian M, Dabbagh A. Effect of intravenous patient controlled ketamine analgesiaon postoperative pain in opium abusers. Anesth Pain Med. 2014;4(1):e14129.

12. Zirak N, Soltani G, Javdani N, Shamloo AS, Bameshki A, Peyvand A. Effect of Fentanyl in Spinal Anesthesia With Bupivacaine in Opium Abusers. Razavi Int J Med. 2014;2(1):e14271.

13. Hassani V, Movassaghi G, Safaian R, Safari S, Zamani MM, Hajiashrafi M, et al. Bupivacaine-sufentanil versus bupivacaine-fen- tanyl in spinal anesthesia of patients undergoing lower extremity surgery. Anesth Pain Med. 2014;4(2):e12091.

14. Bernstein MA, Welch SP. mu-Opioid receptor down-regulation and cAMP-dependent protein kinase phosphorylation in a mouse model of chronic morphine tolerance. Brain Res Mol Brain Res.1998;55(2):237-42.

15. Rogers NF, el-Fakahany E. Morphine-induced opioid receptor down-regulation detected in intact adult rat brain cells. Eur J Pharmacol.1986;124(3):221-30.

16. Mann A, Illing S, Miess E, Schulz S. Different mechanisms of homologous and heterologous mu-opioid receptor phosphorylation. BrJ Pharmacol. 2014.

17. Shannon HE, Lutz EA. Comparison of the peripheral and central effects of the opioid agonists loperamide and morphine in the formalin test in rats. Neuropharmacology. 2002;42(2):253-61.

18. Stein C, Schafer M, Machelska H. Attacking pain at its source: new perspectives on opioids. Nat Med. 2003;9(8):1003-8.

19. Labuz D, Mousa SA, Schafer M, Stein C, Machelska H. Relative contribution of peripheral versus central opioid receptors to antinociception. Brain Res. 2007;1160:30-8.

20. Stein C. Targeting pain and inflammation by peripherally acting opioids. Front Pharmacol. 2013;4:123. 